\title{
Outcomes of Women with Non-Metastatic Triple-Negative Breast Cancer in Oman A single-centre experience
}

"Shiyam Kumar, ${ }^{1}$ Muhammad Furrukh, ${ }^{2}$ Khalid Al-Baimani, ${ }^{1}$ Adil Al-Ajmi, ${ }^{3}$ Ikram A. Burney, Mansour S. Al-Moundhri ${ }^{4}$

$$
\text { نتائج مرضى سرطان الثدي الثلاني السلبي غير المنتشر فئ عمان }
$$

$$
\text { شيام كومار، محمد فروخ، خالد البيماني، عادل العجمي، إكرام برني، منصور المنذري }
$$

ABSTRACT: Objectives: Triple-negative breast cancer (TNBC) is one of the most aggressive and heterogeneous variants of breast cancer. However, little is known regarding the prevalence and outcome of this entity in the Middle East. This study aimed to evaluate the outcomes of TNBC patients at a university hospital in Oman. Methods: This retrospective study took place at the Sultan Qaboos University Hospital, Muscat, Oman, in May 2017. All patients diagnosed with non-metastatic TNBC between December 2000 and December 2015 were included. The patients' electronic medical records were reviewed to identify their clinical and pathological characteristics as well as survival outcomes. Results: A total of 79 patients were diagnosed with non-metastatic TNBC during the study period. The median age was 46 years, with approximately one-third of patients (31.6\%) under 40 years of age. Almost half had an advanced tumour size (49.4\%) or node-positive disease (48.1\%) at presentation and only $16.6 \%$ demonstrated a complete pathological response ( $\mathrm{pCR}$ ) to neoadjuvant chemotherapy. The median survival for all patients was not reached within the study period; however, the median overall survival for stage III patients was 44.6 months. The five-year overall survival for all patients was $64 \%$, increasing to $100 \%$ and $72 \%$ for patients with stage I and II, respectively, and dropping to $47 \%$ for those with stage III disease. Conclusion: The findings of this study indicate that the majority of women with TNBC in Oman present at an advanced stage; moreover, such women have low rates of pCR to neoadjuvant chemotherapy and poor five-year survival.

Keywords: Breast Cancer; Triple-Negative Breast Cancer; Neoadjuvant Therapy; Survival; Patient Outcome Assessment; Oman.

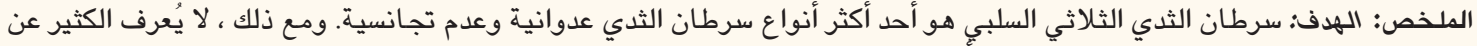

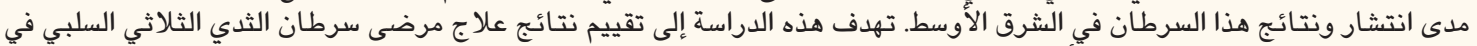

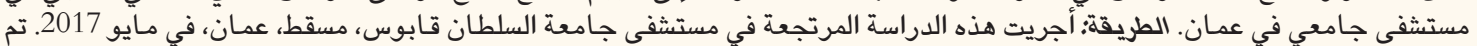

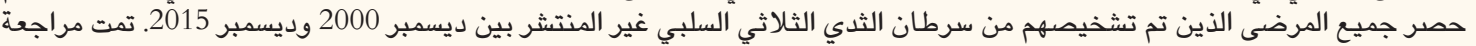

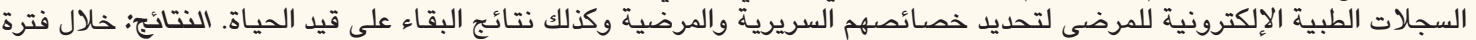

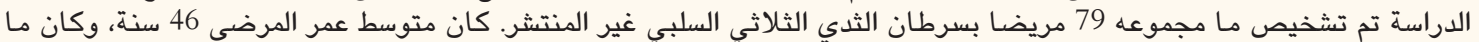

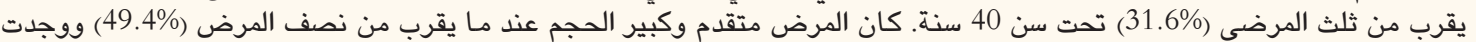

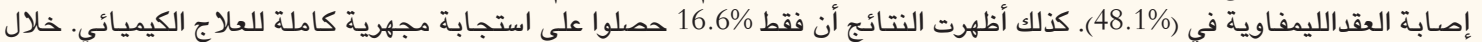

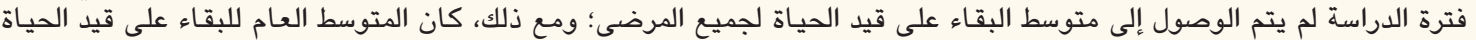

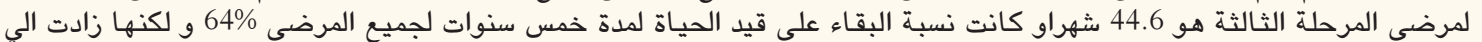

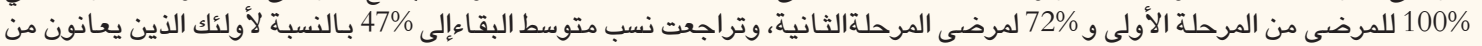

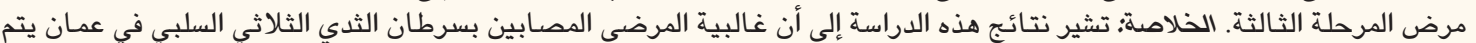

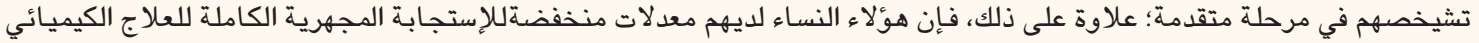

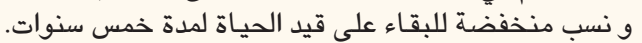

$$
\text { الكلمات المفتاحية: سرطان الثدي؛ سرطان الثدي الثلاثي السلبي؛ العلاج الكيميائي؛ نسب البقاء؛ تقييم نتائج المريض؛ عمان. }
$$

\section{Advances in KNOWLedge}

To the best of the authors' knowledge, this study is the first in Oman to report the clinical presentation, pathological features and outcomes of women diagnosed with triple-negative breast cancer (TNBC). These findings may serve as a reference point for future research on this subject. 


\section{Application to Patient Care}

This study will help clinicians in Oman to evaluate responses to treatment and survival rates according to disease stage among TNBC patients.

Local data may also help health decision-makers to form future policies on patient awareness and TNBC prevention in Oman.

\section{$\mathrm{B}$} REAST CANCER IS A HETEROGENEOUS DISEASE, with variable outcomes based on individual clinical, pathological, molecular and ethnic features. ${ }^{1-4}$ Distinct molecular subtypes of breast cancer and differences in progenitor cells also play a role in disease presentation and prognosis. ${ }^{5}$ Triple-negative breast cancer (TNBC) is a form of breast cancer characterised by a lack of expression of oestrogen, progesterone and human epidermal growth factor receptor 2 (HER2)/ neu receptors, with the diagnosis confirmed by immunohistochemistry and/or other tests such as fluorescence in situ hybridisation. ${ }^{2,6-9}$

Approximately $10-20 \%$ of women with breast cancer in Western countries have TNBC., ${ }^{1,6}$ This variant is considered one of the most aggressive forms of breast cancer, usually presenting at an early age and high grade. It has distinct pathological and molecular markers and variable outcomes as well as an ethnic predilection. ${ }^{2,7-10}$ In general, TNBC cases have poorer outcomes in comparison to other subtypes of breast cancer, despite advances in breast cancer treatment. ${ }^{2,6-9}$

In Oman, government-funded healthcare is provided free of charge to all nationals across the country via a well-established network of primary and secondary health services which are linked with tertiary care hospitals. ${ }^{11}$ Much like the rest of the world, breast cancer is the most common type of cancer among females in Oman; while patients usually present at a younger age and more advanced stage, survival rates are similar to those reported in developed countries.,12 Although there is extensive literature available regarding the outcomes of TNBC cases in Western countries, few studies have been conducted on this topic in the Middle East. Therefore, this study aimed to describe the outcomes of women diagnosed with TNBC and treated at a university hospital in Oman over a 15-year period.

\section{Methods}

This retrospective non-interventional study included all TNBC patients diagnosed and treated at the medical oncology unit of Sultan Qaboos University Hospital (SQUH), Muscat, Oman, between December 2000 and December 2015. Electronic patient records were reviewed from 2006 onwards, as prior to that the hospital utilised a paper-based record system. Patients who were diagnosed earlier than this period and were still alive and being followed-up by the medical oncology unit, were also included in the analysis.

A database was compiled of the patients' clinical and pathological characteristics as well as survival outcomes-including age; gender; clinical stage according to the American Joint Committee on Cancer tumor-node-metastasis (TNM) staging system; final histological subtype, tumour size and grade; Ki67 score; lymph node status; number of lymph nodes retrieved; oestrogen, progesterone and HER2/neu receptor status; surgical and systemic treatment offered; and survival status (i.e. dead or alive) at the time of data collection in May 2017. ${ }^{13}$ All information was retrieved from medical charts as well as radiological and pathological reports. All tissue samples (including those of patients who were diagnosed externally) were reviewed by a pathology team for verification purposes.

Data analysis was performed using the Statistical Package for the Social Sciences (SPSS), Version 20.0 (IBM Corp., Armonk, New York, USA). Kaplan-Meier curves were used to calculate both relapse-free survival (RFS), measured from diagnosis to the date of documented relapse, and overall survival (OS), measured from the date of diagnosis to the date of death or last follow-up. A log-rank test was used for the univariate analysis, while the Cox regression method was applied for the multivariate analysis. A $P$ value of $<0.05$ was considered statistically significant.

This study received ethical approval from the Medical Research \& Ethics Committee of the College of Medicine \& Health Sciences, Sultan Qaboos University (MREC \# 1047).

\section{Results}

A total of 79 female patients were diagnosed with nonmetastatic TNBC during the study period. The median age was 46 years (range: 19-74 years old), with 31.6\% under 40 years old at the time of diagnosis. In total, 73 patients $(92.4 \%)$ were Omani nationals, while the remaining six were from Egypt (2.5\%), Pakistan (2.5\%), South Africa (1.3\%) or India (1.3\%). The majority of patients were premenopausal (65.8\%), had one or more comorbidities (53.2\%) and an above-normal body mass index (65.8\%). Moreover, nearly half had an advanced tumour size of $>5 \mathrm{~cm}$, invading the skin or chest wall (49.4\%) or node-positive disease (48.1\%) at presentation [Table 1]. 
Table 1: Clinical characteristics of women with nonmetastatic triple-negative breast cancer at the Sultan Qaboos University Hospital, Muscat, Oman ( $=79)$

\begin{tabular}{|c|c|}
\hline Characteristic & n (\%) \\
\hline \multicolumn{2}{|l|}{ Age in years } \\
\hline$<40$ & $25(31.6)$ \\
\hline $40-60$ & $46(58.2)$ \\
\hline$>60$ & $8(10.1)$ \\
\hline Median (range) & $46(19-74)$ \\
\hline \multicolumn{2}{|l|}{ Menstrual status } \\
\hline Premenopausal & $52(65.8)$ \\
\hline Menopausal & $27(34.2)$ \\
\hline \multicolumn{2}{|l|}{ Family history of breast cancer } \\
\hline Yes & $5(6.3)$ \\
\hline No & $66(83.5)$ \\
\hline Unknown & $8(10.1)$ \\
\hline \multicolumn{2}{|l|}{ Affected side } \\
\hline Left & $38(48.1)$ \\
\hline Right & $41(51.9)$ \\
\hline \multicolumn{2}{|l|}{ Clinical tumour size } \\
\hline $\mathrm{Tx}$ & $1(1.3)$ \\
\hline $\mathrm{T} 1$ & $6(7.6)$ \\
\hline $\mathrm{T} 2$ & $33(41.8)$ \\
\hline T3 & $23(29.1)$ \\
\hline $\mathrm{T} 4$ & $15(19)$ \\
\hline Inflammatory breast cancer & $1(1.3)$ \\
\hline \multicolumn{2}{|l|}{ Clinical node status } \\
\hline No & $41(51.9)$ \\
\hline N1 & $26(32.9)$ \\
\hline N2 & $11(13.9)$ \\
\hline N3 & $1(1.3)$ \\
\hline \multicolumn{2}{|l|}{ Clinical stage at presentation } \\
\hline I & $5(6.3)$ \\
\hline II & $41(51.9)$ \\
\hline III & $33(41.8)$ \\
\hline
\end{tabular}

$T x=$ tumour size could not be ascertained; $T 1=$ tumour of $\leq 2 \mathrm{~cm} ; T 2=$ tumour of $2.1-5 \mathrm{~cm} ; \mathrm{T3}=$ tumour of $>5 \mathrm{~cm} ;$ T4 = tumour of any size invading the chest wall, breast skin (ulceration or macroscopic nodules) or inflammatory carcinoma; $N O=$ absence of nodal involvement; $N 1$ = metastasis to the ipsilateral axillary lymph nodes that are mobile; $N 2=$ metastasis to the ipsilateral axillary lymph nodes that are fixed; N3 = metastasis to the ipsilateral infra-clavicular lymph nodes with or without axillary lymph nodes.

The median pathological tumour size was $4 \mathrm{~cm}$ (range: $0-13 \mathrm{~cm}$ ). The median number of recovered lymph nodes was three (range: 1-14), with a median of one involved lymph node (range: 0-33). Almost all of the patients had infiltrative ductal carcinomas (97.5\%). The vast majority of tumours were poorly differentiated (i.e. pathological grade of III; 77.2\%) and had a Ki67 proliferation index of $>20 \%$ (74.7\%). In total, 35 women (44.3\%) had a mastectomy and 37 (46.8\%) had breast conservation surgeries. Seven women $(8.9 \%)$ did not undergo surgery, either due to patient refusal (3.8\%), disease progression despite neoadjuvant chemotherapy (NAC; $3.8 \%$ ) or for an unknown reason (1.3\%) [Table 2]. The surgical margins were negative in 39 patients $(49.4 \%)$, with margins of $<1 \mathrm{~mm}$ in four cases (5.1\%), $1-5 \mathrm{~mm}$ in eight cases (10.1\%), 5-10 $\mathrm{mm}$ in four cases (5.1\%) and >10 mm in 23 cases (19.1\%). No information regarding surgical margins was available for the remaining 40 patients (50.6\%).

The majority of patients $(92.4 \%)$ received some type of chemotherapy, with six women (7.6\%) refusing treatment. Overall, NAC therapy was administered to 36 patients (45.6\%), while $37(46.8 \%)$ received adjuvant chemotherapy. A total of 59 patients (74.7\%) were treated with anthracyclines followed by taxanes, while five $(6.3 \%)$ were also treated with platinumbased combination chemotherapy and of those only one patient achieved complete pathological response (pCR). Paclitaxel was administered weekly or thrice weekly, while the two most fit patients were treated with a combination of doxorubicin, docetaxel and cyclophosphamide (TAC). Dose dense doxorubicin and cyclophosphamide (AC) followed by dose dense paclitaxel is not practiced currently at SQUH.

The median number of chemotherapy cycles administered was eight (range: 2-8 and 4-8 cycles for patients receiving adjuvant and NAC regimens, respectively). The main side-effects of first-line treatment were weakness and fatigue (7.6\%), febrile neutropaenia (6.3\%) and non-neutropaenic fever (5.1\%). Of the 36 patients who were treated with NAC, most (69.4\%) received a standard regimen of four cycles of AC, followed by four cycles of docetaxel.

Only six patients (16.6\%) demonstrated pCR to treatment for both primary and nodal disease, while nine patients $(11.4 \%)$ demonstrated a complete response in their recovered lymph nodes which significantly affected the relapse-free survival (RFS) on univariate $\log$ rank analysis $(P=0.029)$. There were 35 cases $(44.3 \%)$ of disease relapse. Of the patients who relapsed, 20 (57.1\%) underwent another biopsy, of which three were diagnosed with hormone-positive disease and were treated with hormonal therapy. Overall, $45.7 \%$ of patients who relapsed were treated with chemotherapy alone, 5.7\% underwent radiotherapy alone and $5.7 \%$ were treated with surgery alone, while 
Table 2: Surgical and pathological characteristics of women with non-metastatic triple-negative breast cancer at the Sultan Qaboos University Hospital, Muscat, Oman (N = 79)

\begin{tabular}{|c|c|}
\hline Characteristic & n (\%) \\
\hline \multicolumn{2}{|l|}{ Type of surgery } \\
\hline Modified radical mastectomy & $31(39.2)$ \\
\hline BCS and ALND & $23(29.1)$ \\
\hline BCS and SLN & $14(17.7)$ \\
\hline Skin-sparing mastectomy and ALND & $3(3.8)$ \\
\hline Simple mastectomy and SLN & $1(1.3)$ \\
\hline None & $7(8.9)$ \\
\hline \multicolumn{2}{|l|}{ Pathological type } \\
\hline Infiltrating ductal carcinoma & $77(97.5)$ \\
\hline Infiltrating lobular carcinoma & $1(1.3)$ \\
\hline Micropapillary carcinoma & $1(1.3)$ \\
\hline \multicolumn{2}{|l|}{ Pathological tumour size } \\
\hline T0 & $8(10.1)$ \\
\hline $\mathrm{T} 1$ & $17(21.5)$ \\
\hline $\mathrm{T} 2$ & $31(39.2)$ \\
\hline T3 & $12(15.2)$ \\
\hline $\mathrm{T} 4$ & $5(6.3)$ \\
\hline Unknown & $6(7.6)$ \\
\hline \multicolumn{2}{|l|}{ Pathological node status } \\
\hline No & $37(46.8)$ \\
\hline Nmic & $1(1.3)$ \\
\hline N1 & $17(21.5)$ \\
\hline N2 & $10(12.7)$ \\
\hline N3 & $7(8.9)$ \\
\hline Unknown & $7(8.9)$ \\
\hline \multicolumn{2}{|l|}{ Pathological stage } \\
\hline pCR to chemotherapy & $6(7.6)$ \\
\hline I & $9(11.4)$ \\
\hline II & $37(46.8)$ \\
\hline III & $20(25.3)$ \\
\hline Unknown & $7(8.9)$ \\
\hline \multicolumn{2}{|l|}{ Pathological grade } \\
\hline I & $2(2.5)$ \\
\hline II & $12(15.2)$ \\
\hline III & $61(77.2)$ \\
\hline Unknown & $4(5.1)$ \\
\hline \multicolumn{2}{|l|}{ Lymphovascular invasion } \\
\hline No & $38(48.1)$ \\
\hline Yes & $24(30.4)$ \\
\hline Unknown & $17(21.5)$ \\
\hline \multicolumn{2}{|l|}{ Ki67 score } \\
\hline$<20 \%$ & $2(2.5)$ \\
\hline$>20 \%$ & $59(74.7)$ \\
\hline Unknown & $18(22.8)$ \\
\hline
\end{tabular}

$B C S=$ breast conservation surgery; $A L N D=$ axillary lymph node dissection; $S L N=\operatorname{sen}-$ tinel lymph node biopsy; TO = main tumour could not be found; $T 1=$ tumour of $\leq 2$ $\mathrm{cm} ; T 2=$ tumour of $2.1-5 \mathrm{~cm} ; \mathrm{T3}=$ tumour of $>5 \mathrm{~cm} ; \mathrm{T4}=$ tumour of any size with invasion or adherence to pectoralis muscle, skin ulceration, skin oedema and inflammatory carcinoma; $N O=$ absence of nodal involvement; $N$ mic $=$ node micrometastases $N 1=$ metastasis to $1-3$ ipsilateral axillary lymph nodes; $N 2=$ metastasis to $4-9$ ipsilateral axillary lymph nodes; $N 3=$ metastasis to $\geq 10$ axillary lymph nodes, infraclavicular lymph nodes; $p C R=$ complete pathological response.

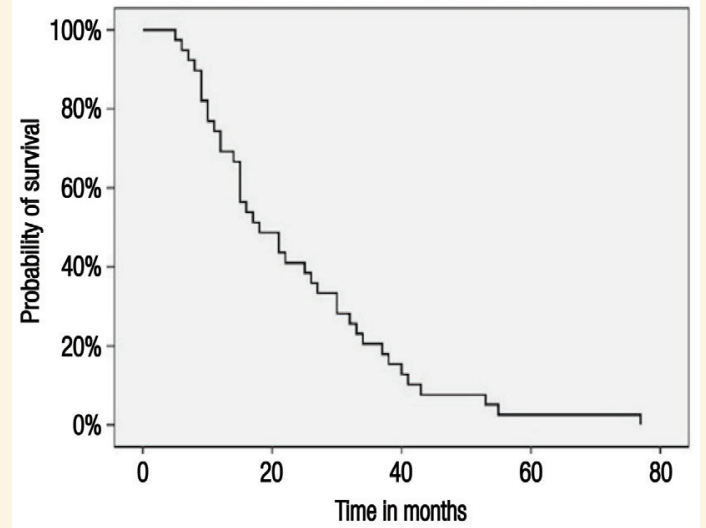

Figure 1: Kaplan-Meier survival curve showing relapsefree survival among women with non-metastatic triplenegative breast cancer at the Sultan Qaboos University Hospital, Muscat, Oman $(\mathrm{N}=79)$.

the remaining $42.8 \%$ of patients were treated with combined treatment modalities.

Out of 29 patients, 13 (44.8\%) received a salvage regimen of gemcitabine and carboplatin for a median of one cycle (range: 0-9 cycles). Growth factors were prescribed in 38 cases $(48.1 \%$ ) as a form of primary or secondary prophylaxis. None of the patients developed treatment-related cardiomyopathy or secondary cancers during the follow-up period. Following chemotherapy and surgery, radiation was administered to 59 patients (74.7\%). At the time of their first local relapse, radiotherapy was incorporated as part of treatment for nine patients (25.7\%). Radiotherapy was administered to five other patients at different intervals as a subsequent line of treatment for recurrent progressive disease.

The median follow-up period was 43 months (range: 0-205 months). By the end of the study period, 44 patients $(55.7 \%)$ were in remission, 25 (31.6\%) had died, four (5.1\%) were alive and undergoing active treatment, one $(1.3 \%)$ was alive and on best supportive care and five (6.3\%) were lost to follow-up. Overall, the vast majority of deaths (96\%) were due to breast cancer, with only one death (1.3\%) occurring due to other causes. The median RFS was 18 months (range: 5-77 months) [Figure 1], with no recurrence noted among stage I patients, while median RFS for stage II and III disease was 27 and 15 months, respectively. The median OS was not reached during the study period [Figure 2A]. The median OS for stage III patients was 44.6 months. Overall, the five-year OS rate was $64 \%$; according to disease stage, five-year survival rates were $100 \%, 72 \%$ and $47 \%$ for patients with stage I, II and III disease, respectively [Figure 2B].

According to a univariate log-rank analysis, a number of factors were found to significantly affect RFS, including age, a positive family history of breast cancer, affected side (i.e. right versus left breast), clinical 

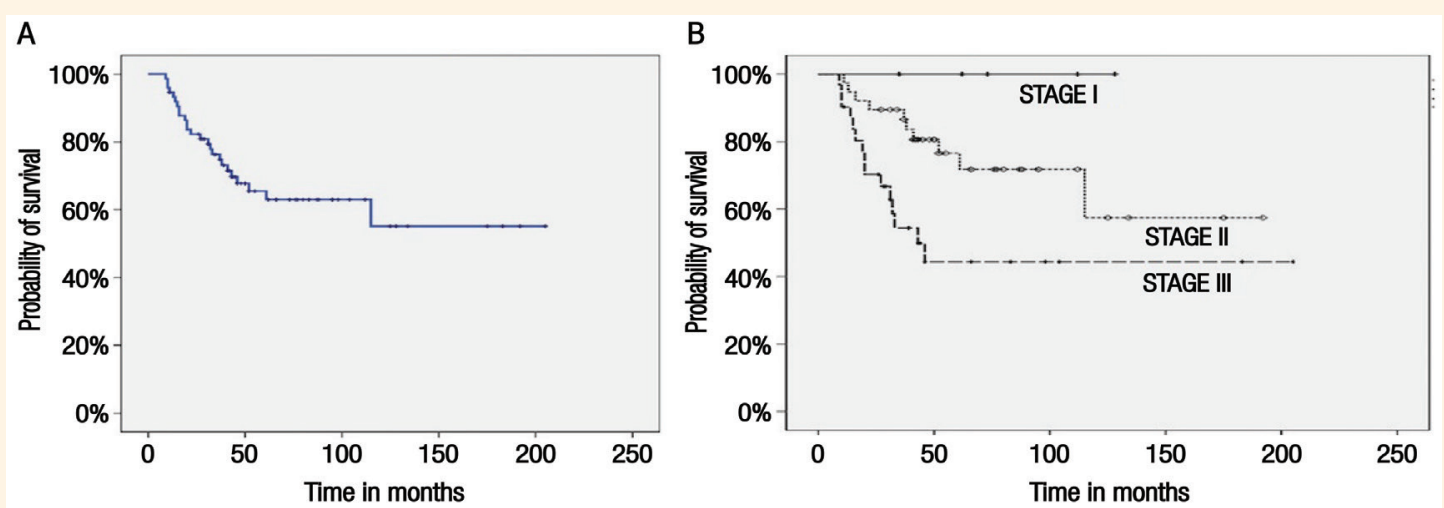

Figure 2: Kaplan-Meier survival curves showing (A) overall survival (OS) and (B) five-year OS according to disease stage among women with non-metastatic triple-negative breast cancer at the Sultan Qaboos University Hospital, Muscat, Oman $(\mathrm{N}=79)$.

Table 3: Univariate analysis of factors affecting median relapse-free and overall survival among women with non-metastatic triple-negative breast cancer at the Sultan Qaboos University Hospital, Muscat, Oman ( $=79)$

\begin{tabular}{|c|c|c|c|c|}
\hline \multirow[t]{2}{*}{ Variable } & \multicolumn{2}{|c|}{ RFS } & \multicolumn{2}{|l|}{ OS } \\
\hline & Median $(95 \% \mathrm{CI})$ & $P$ value & Median $(95 \% \mathrm{CI})^{*}$ & $P$ value \\
\hline $\mathrm{Age}^{+}$ & $3.7(10.6-25.3)$ & $<0.001$ & - & - \\
\hline Family history of breast cancer & $16(12.6-19.3)$ & 0.043 & - & - \\
\hline Affected side & $30(22.8-37.1)$ & 0.016 & - & - \\
\hline Clinical tumour size & $27(18.4-35.5)$ & $<0.001$ & - & - \\
\hline Clinical lymph node status & $18(10.6-25.3)$ & 0.174 & - & - \\
\hline Clinical stage at diagnosis & $27(17.4-36.5)$ & 0.167 & - & - \\
\hline Type of surgery & $11(6.4-15.5)$ & 0.084 & $20(7.2-32.7)$ & $<0.001$ \\
\hline Pathological tumour size & $34(19.5-48.4)$ & $<0.001$ & $61(35-86.9)$ & 0.002 \\
\hline Surgical margins & $32(13.9-50)$ & 0.463 & - & - \\
\hline Pathological node status & $18(12.3-23.6)$ & 0.038 & - & - \\
\hline Lymphovascular invasion & $15(0-33.9)$ & 0.515 & $52(25.4-78.5)$ & 0.041 \\
\hline Ki67 score ${ }^{\dagger}$ & $17(10.7-23.2)$ & $<0.001$ & - & - \\
\hline Chemotherapy regimen & $17(7.9-26)$ & 0.781 & - & - \\
\hline Number of chemotherapy cycles & $15(12.4-17.5)$ & $<0.001$ & - & - \\
\hline Response to NAC & $17(13.7-20.2)$ & $<0.001$ & - & - \\
\hline Adjuvant radiotherapy & $26(12.7-39.2)$ & 0.133 & - & - \\
\hline
\end{tabular}

RFS = relapse-free survival; $O S=$ overall survival; $C I=$ confidence interval; $N A C=$ neoadjuvant chemotherapy.

*95\% CI values for many variables were not available due to a lack of median events. ${ }^{+}$Continuous variable.

tumour size, pathological tumour size, pathological node status, Ki67 score, number of first-line chemotherapy cycles and response to NAC ( $P<0.05$ each). Furthermore, OS was significantly affected by type of surgery, pathological tumour size and lymphovascular invasion $(P<0.05$ each) [Table 3]. However, during a multivariate Cox regression analysis, none of the aforementioned factors were found to be significant in regards to RFS; only pathological tumour size was found to significantly affect OS.

\section{Discussion}

In Oman, TNBC accounts for $18.8 \%$ of all female breast cancers. ${ }^{4}$ However, to the best of the authors' knowledge, no previous studies have reported the outcomes of clinical or pathological features of TNBC cases. In the current study, the median age at presentation was 46 years, which is similar to that reported by other researchers in Oman as well as other parts of the world. ${ }^{1,3,14-16}$ However, the percentage of patients 
in Oman with stage I disease at presentation (6.3\%) was considerably lower than reports from the rest of the world, even in comparison to the low percentage of African American women presenting with stage I disease in the USA. ${ }^{10,17}$ This may be a regional trend as only $3.9 \%$ and $10.8 \%$ of women from India and Pakistan, respectively, have been reported to have stage I disease at presentation. ${ }^{3,18}$

Worldwide, tumour size and lymph node status remain significant prognostic factors in TNBC cases, despite the availability of numerous molecular markers, and are an integral part of the American Joint Committee on Cancer TNM staging system. ${ }^{13}$ Clinically, almost half of the patients in the current cohort presented with an advanced tumour size (T3 or T4), nodepositive disease and grade III disease. Bonsang-Kitzis et al. reported similar results in France. ${ }^{14}$ Furthermore, the current study found a significant correlation between tumour size and positive lymph node status, with both factors prognostic for RFS and OS.

Nevertheless, although it has been suggested that tumour size may predict lymph node involvement, this may not necessarily be the case; in contrast to hormone-positive breast cancer, the proliferation rate of TNBC cells is high and there is a high probability of lymph node involvement, independent of tumour size. ${ }^{19}$ Foulkes et al. found no correlation between tumour size and positive lymph node status among carriers of the breast cancer (BRCA) 1 gene, although there was a highly significant correlation between these variables among BRCA2 carriers and both $B R C A 1$ and $B R C A 2$ non-carriers. ${ }^{20}$

Overall, pCR to treatment (i.e. the absence of residual disease in the primary site as well in the recovered lymph nodes) is an important endpoint when determining NAC treatment efficacy for advanced stage non-metastatic breast cancer; moreover, this variable has been associated with improved diseasefree survival (DFS) and OS. ${ }^{14}$ In the present study, the post-NAC pCR rate was significantly lower in comparison to those reported from Western countries, despite the use of similar chemotherapy agents (i.e. anthracyclines and taxanes). ${ }^{14,21-23}$ This may be due to the fact that most patients in the current study presented at an advanced stage; in comparison to a report from France, 93.7\% versus 26\% of patients presented at grades I or II. ${ }^{14}$ Moreover, $14.9 \%$ of patients from the USA had T3 tumours at presentation, while $29.1 \%$ and 19\% of patients had T3 and T4 tumours, respectively, before starting NAC. ${ }^{21}$ Other factors contributing to this difference in findings could be the small sample size of the present cohort or biological differences in disease subtype. ${ }^{24}$
In the current study, clinical and pathological tumour size and pathological lymph node status significantly affected RFS for all patients; however, for patients who were treated with NAC, only pathological lymph node status was associated significantly with RFS $(P=0.029)$. This finding contradicts those previously reported by Bonsang-Kitzis et al. in which post-NAC residual disease in the primary site as well as the axillary lymph nodes was associated with poor DFS and post-NAC pCR in primary disease was associated with improved OS. ${ }^{14}$

Generally, anthracyclines and taxanes (either in sequence or combination) in conventional or dosedense fashion are considered the standard of care internationally for breast cancer patients in either an adjuvant or neoadjuvant setting. ${ }^{22,25}$ Chemotherapy alone remains the proven option for TNBC patients as hormonal agents or anti-HER2-directed therapy cannot be administered. ${ }^{26}$ The majority of patients in the present study were treated with four cycles of $\mathrm{AC}$, followed by four cycles of docetaxel. Other prescribed regimens included paclitaxel (on a weekly as well as thrice weekly basis), carboplatin along with docetaxel after AC and TAC. However, none of the patients were treated on a dose-dense schedule. While the use of platinum compounds has resulted in significantly improved $\mathrm{pCR}$ rates, especially among TNBC patients harbouring BRCA1 mutations in a neoadjuvant setting, this treatment is not yet routinely used. ${ }^{6,26}$ Only five out of 36 patients in the present study received carboplatin in neoadjuvant setting; of these, only one (13.9\%) achieved pCR.

In light of lower response rates to conventional chemotherapeutic agents, there is increasing interest in newer treatment modalities. Poly (adenosine diphosphate-ribose) polymerase inhibitors are an option for $B R C A$ mutation-associated cancers, including TNBC. ${ }^{26}$ Recently, a phase III trial showed improved efficacy among patients with $B R C A$ germline-mutant metastatic breast cancer. ${ }^{27} \mathrm{~A}$ phase II trial of metastatic TNBC patients found that pembrolizumab as a single agent had an overall response rate of only 5\%; in contrast, immune-modulating agents such as checkpoint inhibitors have shown significantly better results in combination with chemotherapy. ${ }^{26,28}$ Another phase II trial investigating pembrolizumab combined with anthracyclines and taxanes in a neoadjuvant setting showed remarkable pCR rates of up to $71.4 \%$ in patients with TNBC. ${ }^{29}$

Generally, OS rates are poorer among TNBC patients in comparison to those with other breast cancer subtypes. ${ }^{1,7,9,21,25,26}$ In the USA, the five-year OS rate is $64-80 \%$ among patients with TNBC; this is higher 
than that reported in the current cohort (63\%). ${ }^{1,21,30}$ Surprisingly, higher RFS and OS rates have been reported among patients with TNBC from India and Pakistan as well., ${ }^{3,18}$ Despite access to standard treatment modalities, one possible reason for the lower survival rate in the current study may be ethnic differences which can affect breast cancer-related outcomes. ${ }^{1,17}$ Stage by stage, the five-year OS rate for the present cohort was better in comparison to that of African American women, but worse than that of Caucasian and South Asian women., ${ }^{1,3,17,18}$ Various molecular subtypes of TNBC (i.e. basal-like [BL]1, BL2, mesenchymal and luminal androgen receptor subtypes) have also shown different responses to treatment and associated outcomes. ${ }^{6,7,9,26}$ As such, it is possible that patients in Oman have a divergent molecular subtype of TNBC resulting in more aggressive disease behaviour and poor survival. While it is difficult to generalise the results of this cohort to the entire country, such trends have been reported previously. ${ }^{4,12}$

The retrospective nature of the study, the small sample size and that the analysis was spread over a long period of time are the main limitations of this study. Despite these limitations, this study provides valid data regarding presentation stage, treatment offered and outcome of Omani patients and also discuss the possibilities of poorer associated outcomes. This study also can serve as a scaffolding for future studies in TNBC which should also assess the molecular signatures of different subtypes of breast cancer in Arab women and the differences in outcome.

\section{Conclusion}

The current study found that women with TNBC in Oman presented at a younger age in comparison to Western countries. In addition, almost half of the patients presented with large tumours of high grades as well as early lymph node metastasis. Moreover, pCR after completion of NAC was rare and the OS rate was poor. This is likely due to the fact that patients presented at a more advanced stage. In addition, ethnic and biological differences in terms of molecular TNBC subtypes may also play a role. Further molecular studies are recommended to confirm this hypothesis.

\section{CONFLICT OF INTEREST}

The authors declare no conflicts of interest.

\section{FUNDING}

No funding was received for this study.

\section{References}

1. Bauer KR, Brown M, Cress RD, Parise CA, Caggiano V. Descriptive analysis of estrogen receptor (ER)-negative, progesterone receptor (PR)-negative, and HER2-negative invasive breast cancer, the so-called triple-negative phenotype: A populationbased study from the California Cancer Registry. Cancer 2007; 109:1721-8. https://doi.org/10.1002/cncr.22618.

2. Brewster AM, Chavez-MacGregor M, Brown P. Epidemiology, biology, and treatment of triple-negative breast cancer in women of African ancestry. Lancet Oncol 2014; 15:e625-34. https://doi.org/10.1016/S1470-2045(14)70364-X

3. Gogia A, Raina V, Deo SV, Shukla NK, Mohanti BK. Triplenegative breast cancer: An institutional analysis. Indian J Cancer 2014; 51:163-6. https://doi.org/10.4103/0019-509X.138275.

4. Kumar S, Burney IA, Al-Ajmi A, Al-Moundhri MS. Changing trends of breast cancer survival in Sultanate of Oman. J Oncol 2011; 2011:316243. https://doi.org/10.1155/2011/316243.

5. Prat A, Perou CM. Mammary development meets cancer genomics. Nat Med 2009; 15:842-4. https://doi.org/10.1038/ nm0809-842.

6. Carey L, Winer E, Viale G, Cameron D, Gianni L. Triplenegative breast cancer: Disease entity or title of convenience? Nat Rev Clin Oncol 2010; 7:683-92. https://doi.org/10.1038/ nrclinonc.2010.154.

7. Kandil D, Khan A. Triple negative breast carcinoma: The good, the bad and the ugly. Diagn Histopathol (Oxf) 2012; 18:210-16. https://doi.org/10.1016/j.mpdhp.2012.02.001.

8. Carey LA. Directed therapy of subtypes of triple-negative breast cancer. Oncologist 2011; 16:71-8. https://doi.org/10.1634/the oncologist.2011-S1-71.

9. Foulkes WD, Smith IE, Reis-Filho JS. Triple-negative breast cancer. N Engl J Med 2010; 363:1938-48. https://doi.org/10.1 056/NEJMra1001389.

10. Trivers KF, Lund MJ, Porter PL, Liff JM, Flagg EW, Coates RJ, et al. The epidemiology of triple-negative breast cancer, including race. Cancer Causes Control 2009; 20:1071-82. https://doi. org/10.1007/s10552-009-9331-1.

11. World Health Organization Regional Health Systems Observatory. Health system profile: Oman. From: http:// apps.who.int/medicinedocs/documents/s17304e/s17304e.pdf Accessed: Feb 2019.

12. Al-Moundhri M, Al-Bahrani B, Pervez I, Ganguly SS, Nirmala V, Al-Madhani A, et al. The outcome of treatment of breast cancer in a developing country--Oman. Breast 2004; 13:139-45. https://doi.org/10.1016/j.breast.2003.10.001.

13. American Joint Commitee on Cancer. What is cancer staging? From: www.cancerstaging.org/references-tools/Pages/What-isCancer-Staging.aspx Accessed: Feb 2019.

14. Bonsang-Kitzis H, Chaltier L, Belin L, Savignoni A, Rouzier R, Sablin MP, et al. Beyond axillary lymph node metastasis, BMI and menopausal status are prognostic determinants for triplenegative breast cancer treated by neoadjuvant chemotherapy. PLoS One 2015; 10:e0144359. https://doi.org/10.1371/journal. pone.0144359.

15. Mehdi I, Monem EA, Al Bahrani BJ, Al Kharusi S, Nada AM, Al Lawati J, et al. Age at diagnosis of female breast cancer in Oman: Issues and implications. South Asian J Cancer 2014; 3:101-6. https://doi.org/10.4103/2278-330X.130442.

16. Rahman M, Ahsan A, Begum F, Rahman K. Epidemiology, risk factors and tumor profiles of breast cancer in Bangladeshi underprivileged women. Gulf J Oncolog 2015; 1:34-42.

17. Field TS, Buist DS, Doubeni C, Enger S, Fouayzi H, Hart G, et al. Disparities and survival among breast cancer patients. J Natl Cancer Inst Monogr 2005:88-95. https://doi.org/10.1093/ jncimonographs/lgi044. 
18. Bhatti AB, Khan AI, Siddiqui N, Muzaffar N, Syed AA, Shah MA, et al. Outcomes of triple-negative versus non-triple-negative breast cancers managed with breast-conserving therapy. Asian Pac J Cancer Prev 2014; 15:2577-81. https://doi.org/10.7314/ apjcp.2014.15.6.2577.

19. Foulkes WD, Reis-Filho JS, Narod SA. Tumor size and survival in breast cancer--A reappraisal. Nat Rev Clin Oncol 2010; 7:348-53. https://doi.org/10.1038/nrclinonc.2010.39.

20. Foulkes WD, Metcalfe K, Hanna W, Lynch HT, Ghadirian P, Tung N, et al. Disruption of the expected positive correlation between breast tumor size and lymph node status in BRCA1related breast carcinoma. Cancer 2003; 98:1569-77. https://doi. org/10.1002/cncr.11688.

21. Liedtke C, Mazouni C, Hess KR, André F, Tordai A, Mejia JA, et al. Response to neoadjuvant therapy and long-term survival in patients with triple-negative breast cancer. J Clin Oncol 2008; 26:1275-81. https://doi.org/10.1200/JCO.2007.14.4147.

22. von Minckwitz G, Martin M. Neoadjuvant treatments for triple-negative breast cancer (TNBC). Ann Oncol 2012; 23:vi35-9. https://doi.org/10.1093/annonc/mds193.

23. von Minckwitz G, Untch M, Blohmer JU, Costa SD, Eidtmann H, Fasching PA, et al. Definition and impact of pathologic complete response on prognosis after neoadjuvant chemotherapy in various intrinsic breast cancer subtypes. J Clin Oncol 2012; 30:1796-804. https://doi.org/10.1200/ JCO.2011.38.8595.

24. Rouzier R, Perou CM, Symmans WF, Ibrahim N, Cristofanilli M, Anderson K, et al. Breast cancer molecular subtypes respond differently to preoperative chemotherapy. Clin Cancer Res 2005; 11:5678-85. https://doi.org/10.1158/1078-0432.CCR-04-2421.
25. Mustacchi G, De Laurentiis M. The role of taxanes in triplenegative breast cancer: Literature review. Drug Des Devel Ther 2015; 9:4303-18. https://doi.org/10.2147/DDDT.S86105.

26. Mayer IA, Dent R, Tan T, Savas P, Loi S. Novel targeted agents and immunotherapy in breast cancer. Am Soc Clin Oncol Educ Book 2017; 37:65-75. https://doi.org/10.14694/EDBK_175631.

27. Robson M, Im SA, Senkus E, Xu B, Domchek SM, Masuda N, et al. Olaparib for metastatic breast cancer in patients with a germline BRCA mutation. N Engl J Med 2017; 377:523-33. https://doi.org/10.1056/NEJMoa1706450.

28. Adams S, Schmid P, Rugo HS, Winer EP, Loirat D, Awada A, et al. Phase 2 study of pembrolizumab (pembro) monotherapy for previously treated metastatic triple-negative breast cancer (mTNBC): KEYNOTE-086 cohort A. J Clin Oncol 2017; 35:S1008. https://doi.org/10.1200/JCO.2017.35.15_suppl.1008.

29. Nanda R, Liu MC, Yau C, Asare S, Hylton N, Veer LV, et al. Pembrolizumab plus standard neoadjuvant therapy for highrisk breast cancer (BC): Results from I-SPY 2. J Clin Oncol 2017; 35:S506. https://doi.org/10.1200/JCO.2017.35.15_suppl.506.

30. Haffty BG, Yang Q, Reiss M, Kearney T, Higgins SA, Weidhaas J, et al. Locoregional relapse and distant metastasis in conservatively managed triple negative early-stage breast cancer. J Clin Oncol 2006; 24:5652-7. https://doi.org/10.1200/ JCO.2006.06.5664. 